\title{
Etude théorique et expérimentale des propriétés gyrométriques d'un oscillateur à mélange à quatre ondes en anneau
}

\author{
M. Pinard, M. Vallet et G. Grynberg \\ Laboratoire de Spectroscopie Hertzienne de l'E.N.S., Université Pierre et Marie Curie, Tour 12, \\ 75252 Paris Cedex 05
}

(Reçu le 22 mars 1990, accepté le 6 juin 1990)

\begin{abstract}
Résumé. - Nous étudions les propriétés d'un oscillateur en anneau utilisant le mélange à quatre ondes dans une vapeur atomique comme processus de gain. Notre étude concerne ici le mode de fonctionnement quasi dégénéré. L'étude théorique est faite au moyen des équations de Lamb. Nous montrons que le système se comporte comme un gyromètre et que son facteur d'échelle est identique à celui des gyrolasers. Nous calculons la zone aveugle de ce gyromètre, zone aveugle qui est due à la rétrodiffusion sur les miroirs. Nous montrons aussi que l'utilisation d'une nonlinéarité intrinsèque permet de s'affranchir du problème de la zone aveugle par un moyen purement optique. Finalement nous décrivons les expériences faites en utilisant une vapeur de sodium qui nous ont permis de mettre en évidence ces propriétés gyrométriques.
\end{abstract}

\begin{abstract}
We present a theoretical and experimental study of the steady-state regime of a ring four-wave mixing oscillator. We study here the properties of the quasi-degenerate mode. The theory is developped using the Lamb equations. We show that the four-wave mixing oscillator can be used as a gyrometer and that its scale factor is identical to the gyrolaser scale factor. We calculate the lock-in band, which is due to backscattering on the mirrors, and show that an intrinsic non-linearity can be used to circumvent this problem by optical means only. Finally we describe the experiments we have performed using sodium vapor to observe these gyrometric properties.
\end{abstract}

\section{Introduction.}

L'étude du mélange à quatre ondes a suscité beaucoup d'intérêt depuis plusieurs années. Cet intérêt est stimulé à la fois par les applications possibles en conjugaison de phase [1] et par la possibilité de générer soit des états comprimés du rayonnement [2-6], soit des faisceaux de photons jumeaux [7]. De nombreuses études ont porté sur les propriétés de l'auto-oscillation de cavité utilisant le mélange à quatre ondes comme processus générant les photons. Toutefois la plupart des études réalisées avec des vapeurs atomiques utilisent le milieu conjuguant la phase comme miroir amplificateur [8]. Nous nous intéressons dans cet article à l'étude de l'auto-oscillation d'une cavité en anneau où le milieu conjuguant la phase est utilisé comme amplificateur et est placé dans un des bras de la cavité. Dans ce domaine, de nombreux résultats ont déjà été obtenus dans les milieux photoréfractifs [9]. Dans notre 
étude, le milieu non linéaire, mélangeant les ondes est constitué par une vapeur atomique. Nous avons montré récemment que les résultats obtenus dans le cas des solides photoréfractifs ne s'appliquent pas toujours aux vapeurs atomiques. Par exemple, dans le cas d'un solide photoréfractif, la fréquence de l'oscillation diffère légèrement de la fréquence des pompes [10-14] tandis que dans le cas d'une vapeur de sodium pure les deux fréquences généralement coïncident [15-18].

En fait, l'analyse des résultats expérimentaux [17] montrent que l'oscillation dans le cas d'une vapeur de sodium pure a des propriétés similaires à celles d'un oscillateur paramétrique dégénéré [19]. Il existe néanmoins des différences entre les deux dispositifs, nous avons par exemple observé que l'oscillation est presque toujours bistable dans le cas du mélange à quatre ondes [17] tandis qu'une telle propriété n'est pas observée dans le cas d'un oscillateur paramétrique dégénéré.

Pour étudier théoriquement les propriétés de l'oscillation d'une cavité utilisant une vapeur atomique conjuguant la phase comme amplificateur, nous utilisons la méthode de Lamb [20]. Nous l'appliquons ici à l'étude d'un oscillateur en anneau. Après avoir décrit le modèle (\$ 2), nous étudions, dans le paragraphe 3 l'influence des collisions sur le fonctionnement quasi dégénéré. Nous montrons qu'en présence de collisions et pour des ondes pompes d'intensité différente il est possible de lever la dégénérescence entre les fréquences des deux ondes contrarotatives. Dans le paragraphe 4 nous décrivons l'expérience qui nous a permis de mettre en évidence cette propriété et de tracer la réponse gyrométrique d'un oscillateur paramétrique atomique. Dans le paragraphe 5 nous décrivons les résultats obtenus en utilisant pour lever la dégénérescence une méthode plus conventionnelle, cette méthode consistant à placer dans la cavité un rotateur de Faraday. Il faut aussi souligner que l'étude théorique que l'on fait ici peut avoir des applications dans le cas d'autres dispositifs gyrométriques où le mélange à quatre ondes se manifeste comme un effet secondaire [21].

\section{Description du modèle.}

L'oscillateur étudié est constitué d'une cavité en anneau, cette cavité contient un milieu non linéaire qui la remplit totalement, ce milieu non linéaire interagit avec deux ondes pompes $\mathbf{E}_{1}$ et $\mathbf{E}_{2}$ se propageant en sens opposé. On posera $\mathbf{E}_{\mathrm{p}}=\mathbf{E}_{1}+\mathbf{E}_{2}$. Le milieu non linéaire est une vapeur atomique qui est décrite par un ensemble d'atomes à deux niveaux a et b (Fig. 1). La fréquence atomique est $\omega_{0}$ et $N$ est le nombre d'atomes par unité de volume. L'élément de matrice de l'opérateur dipolaire électrique entre les deux niveaux est $d$. Les deux faisceaux pompes $\mathbf{E}_{1}$ et $\mathbf{E}_{2}$ ont même fréquence $\omega$ et même polarisation linéaire $\mathbf{e}_{\mathbf{x}}$. On suppose que

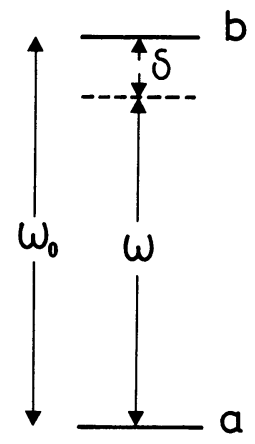

Fig. 1. - Schéma du système atomique à deux niveaux.

[Scheme of the two level atomic system.] 
l'excitation est non résonante, le désaccord $\delta=\omega_{0}-\omega$ entre la fréquence atomique et la fréquence des pompes est beaucoup plus grand que la largeur naturelle $\gamma_{\mathrm{ab}}$ et que la largeur Doppler $k u$. Les fréquences de Rabi résonantes des atomes dans les ondes pompes sont supposées très petites devant le désaccord $|\delta|$. Nous sommes ainsi dans une situation où un calcul perturbatif peut être utilisé pour calculer la réponse atomique. La méthode utilisée consiste à supposer connu le champ intra-cavité $E_{\mathrm{c}}(\mathbf{r}, t)$ puis à calculer la polarisation atomique $\mathbf{P}(\mathbf{r}, t)$ induite par le champ total $E(\mathbf{r}, t)=E_{\mathrm{c}}(\mathbf{r}, t)+E_{\mathrm{p}}(\mathbf{r}, t)$ interagissant avec les atomes. En utilisant la polarisation $\mathbf{P}(\mathbf{r}, t)$ comme terme source dans les équations de Maxwell on calcule le champ rayonné par les atomes et en particulier le champ rayonné dans la cavité. La condition d'auto-cohérence de ce calcul impose que ce dernier champ coïncide avec $\mathbf{E}_{\mathrm{c}}$.

Dans toute la suite, nous supposons que tous les champs ont la même polarisation linéaire $\mathbf{e}_{\mathrm{x}}$, par conséquent nous omettons cette polarisation et raisonnons comme si ces champs étaient scalaires. Le champ pompe total s'écrit :

$$
\begin{gathered}
E_{\mathrm{p}}(\mathbf{r}, t)=\mathcal{R e} \sum_{j=1}^{2} \varepsilon_{\mathrm{j}} \mathrm{e}^{-i \omega_{\mathrm{j}} t} \mathrm{e}^{i \mathbf{k}_{\mathrm{j}} \cdot \mathbf{r}}=E_{\mathrm{p}}^{+}(\mathbf{r}, t)+\text { c.c. } \\
\omega_{1}=\omega_{2}=\omega \\
\mathbf{k}_{1}=-\mathbf{k}_{2} .
\end{gathered}
$$

avec

Nous supposons que le champ intra-cavité est une superposition linéaire d'un petit nombre de modes propres spatiaux de la cavité. La composante de fréquence positive de $E_{\mathrm{c}}(\mathbf{r}, t)$ s'écrit donc :

$$
\begin{aligned}
& E_{\mathrm{c}}(\mathbf{r}, t)=\mathscr{R e} \sum_{n} \delta_{\mathrm{n}}(t) \mathrm{e}^{-i \omega_{\mathrm{n}} t} U_{\mathrm{n}}(z)=E_{\mathrm{c}}^{+}(\mathbf{r}, t)+\text { c.c. } \\
& \frac{\partial^{2}}{\partial z^{2}} U_{\mathrm{n}}(z)+\Omega_{\mathrm{n}}^{2} U_{\mathrm{n}}(z)=0 .
\end{aligned}
$$

avec

Dans le cas d'une cavité en anneau immobile de longueur $L$ les modes propres normés s'écrivent :

avec

$$
\begin{aligned}
U_{\mathrm{n}}(z) & =\exp \pm i k_{\mathrm{n}} z \\
k_{\mathrm{n}} & =n \frac{2 \pi}{L} .
\end{aligned}
$$

L'ensemble des champs appliqués va créer dans le milieu une polarisation $P(\mathbf{r}, t)$ qui s'écrit :

$$
P(\mathbf{r}, t)=P^{+}(\mathbf{r}, t)+\text { c.c. }
$$

Cette polarisation qui est non linéaire contient deux types de composantes: des composantes dont la phase totale coïncide avec celle d'un des champs appliqués et des composantes dont la phase totale ne coïncide avec aucune des phases des ondes appliquées. Les composantes de $P(\mathbf{r}, t)$ du second type sont responsables de l'émission d'onde dans des directions qui ne coïncident ni avec les directions de la cavité ni avec la direction des ondes pompes. On ne tiendra pas compte de ces dernières composantes car on peut montrer que leurs contributions sont négligeables.

La dépendance spatiale du champ intra-cavité étant connue on peut établir l'équation d'évolution temporelle des $\boldsymbol{E}_{\mathrm{n}}(t)$. Ces équations d'évolution s'obtiennent à partir des équations de Maxwell appliquées au champ total $\mathbf{E}$, qui s'écrivent :

$$
-\frac{\partial^{2}}{\partial z^{2}} E+\frac{1}{c^{2}} \frac{\partial^{2}}{\partial t^{2}} E+\mu_{0} \cdot \sigma \frac{\partial E}{\partial t}=-\mu_{0} \cdot \frac{\partial^{2} P}{\partial t^{2}} .
$$


Pour éviter un problème délicat de conditions aux limites, nous remplaçons comme dans la théorie traditionnelle de Lamb du laser les pertes dues à la transmission des miroirs par des pertes dues à la présence d'un milieu conducteur de conductivité $\sigma$, ce milieu étant supposé uniforme et remplissant toute la cavité.

L'équation d'évolution de $\varepsilon_{\mathrm{n}}(t)$ est obtenue en multipliant l'équation (5) par $c^{2} U_{\mathrm{n}}^{*}(z) \mathrm{e}^{i \omega_{\mathrm{n}} t}$ et en intégrant spatialement le résultat sur un volume de longueur $L$ et de dimensions transversales grandes devant le pas des réseaux créés par les ondes lumineuses. On obtient alors :

$$
\left(\Omega_{\mathrm{n}}^{2}-\omega_{\mathrm{n}}^{2}\right) \varepsilon_{\mathrm{n}}-i \omega_{\mathrm{n}} \frac{\sigma}{\varepsilon_{0}} \varepsilon_{\mathrm{n}}-2 i \omega_{\mathrm{n}} \dot{\varepsilon}_{\mathrm{n}}=+\frac{\omega_{\mathrm{n}}^{2}}{\varepsilon_{0}} \mathfrak{T}_{\mathrm{n}}
$$

avec

$$
\mathcal{T}_{\mathrm{n}}=\frac{2 \int_{v} P^{+}(\mathrm{r}, t) \mathrm{e}^{i \omega_{\mathrm{n}} t} U_{\mathrm{n}}^{*}(z) \mathrm{d}^{3} r}{\int_{v}\left|U_{\mathrm{n}}(z)\right|^{2} \mathrm{~d}^{3} r}
$$

Comme la différence $\left|\Omega_{\mathrm{n}}-\omega_{n}\right|$ est toujours très petite devant $\omega_{\mathrm{n}}$ l'équation précédente se simplifie, on obtient :

$$
\left[\left(+\Omega_{\mathrm{n}}-\omega_{\mathrm{n}}\right)-\frac{i}{2} \frac{\sigma}{\varepsilon_{0}}\right] \mathcal{E}_{\mathrm{n}}-i \dot{\varepsilon}_{\mathrm{n}}=\frac{\omega_{\mathrm{n}}}{2 \varepsilon_{0}} \mathfrak{T}_{\mathrm{n}} .
$$

En décomposant $\varepsilon_{\mathrm{n}}$ en fonction de son amplitude $E_{\mathrm{n}}$ et de sa phase $\varphi_{\mathrm{n}}$ :

$$
\varepsilon_{\mathrm{n}}=E_{\mathrm{n}} \mathrm{e}^{-i \varphi_{\mathrm{n}}}
$$

On en déduit les équations d'évolution de $E_{\mathrm{n}}$ et de $\varphi_{\mathrm{n}}$ :

$$
\begin{gathered}
\dot{E}_{\mathrm{n}}+\frac{\sigma}{2 \varepsilon_{0}} E_{\mathrm{n}}=-\frac{\omega_{\mathrm{n}}}{2 \varepsilon_{0}} \operatorname{Im}\left(\mathfrak{T}_{\mathrm{n}} \mathrm{e}^{i \varphi_{\mathrm{n}}}\right) \\
-\Omega_{\mathrm{n}}+\left(\omega_{\mathrm{n}}+\dot{\varphi}_{\mathrm{n}}\right)=-\frac{\omega_{\mathrm{n}}}{2 \varepsilon_{0}} \frac{1}{E_{\mathrm{n}}} \mathcal{R e}\left(\mathfrak{T}_{\mathrm{n}} \mathrm{e}^{i \varphi_{\mathrm{n}}}\right) .
\end{gathered}
$$

On voit que si on connaît les composantes $\mathfrak{T}_{\mathrm{n}}$ en fonction des $\boldsymbol{\varepsilon}_{\mathrm{n}}$ et des $\boldsymbol{\varepsilon}_{\mathrm{j}}$, on en déduit les équations d'auto-cohérence du champ intra-cavité. Notre tâche consiste donc premièrement à exprimer les amplitudes $\mathfrak{T}_{\mathrm{n}}$ en fonction des $\varepsilon_{\mathrm{n}}$ et des $\varepsilon_{\mathrm{j}}$ puis à résoudre les équations d'évolution ainsi obtenues. Pour trouver la valeur des champs intra-cavité en régime stationnaire, il faut également résoudre les équations d'évolution des champs pompes $\boldsymbol{E}_{1}$ et $\boldsymbol{E}_{2}$. A priori les amplitudes $\varepsilon_{1}$ et $\varepsilon_{2}$ dépendent simultanément de $z$ et de $t$. En faisant l'hypothèse de l'enveloppe lentement variable, on obtient :

$$
\begin{aligned}
& \left(k^{2}-\frac{\omega^{2}}{c^{2}}\right) \mathcal{E}_{1}-2 i k \frac{\partial \mathcal{E}_{1}}{\partial z}-2 i \frac{\omega}{c^{2}} \frac{\partial \mathcal{E}_{1}}{\partial t}=+\mu_{0} \omega^{2} \mathfrak{T}_{1} \\
& \left(k^{2}-\frac{\omega^{2}}{c^{2}}\right) \mathcal{E}_{2}+2 i k \frac{\partial \mathcal{E}_{2}}{\partial z}-2 i \frac{\omega}{c^{2}} \frac{\partial \mathcal{E}_{2}}{\partial t}=+\mu_{0} \omega^{2} \mathfrak{T}_{2}
\end{aligned}
$$

avec

$$
\mathfrak{T}_{\mathrm{j}}=\frac{2}{V} \int_{V} P^{+}(\mathbf{r}, t) \mathrm{e}^{i \omega_{\mathrm{j}} t} \mathrm{e}^{-i \mathbf{k}_{\mathrm{j}} \cdot \mathbf{r}} \mathrm{d}^{3} r \quad(j=1,2)
$$


Dans les équations (9.c) et (9.d) le terme dû aux pertes ne figure pas car les ondes pompes ne circulent pas dans la cavité, leur direction faisant un petit angle $\theta$ avec l'axe de celle-ci. Les dimensions du volume $V$ sont grandes devant le pas des différents réseaux mais petites devant les dimensions spatiales de variation des $\varepsilon_{\mathrm{j}}(j=1,2)$.

\section{Cavité en anneau, fonctionnement quasi dégénéré. Influence des collisions.}

A) Position DU PROBlÈME. - Dans ce paragraphe, on considère un cas où le champ intracavité est la superposition de deux ondes planes contrarotatives. On s'intéresse à un mode de fonctionnement quasi dégénéré, c'est-à-dire que lorsque la cavité est immobile on a :

$$
U_{3}(z)=U_{4}^{*}(z)=\exp i k z
$$

avec

$$
k=n \frac{c}{L}=\frac{\Omega}{c} .
$$

Lorsque la cavité tourne, sa longueur dépend du sens dans lequel la lumière la parcourt. On voit que cette différence lève la dégénérescence des fréquences propres pour deux ondes contrarotatives. Cette levée de dégénérescence due à l'effet Sagnac [22-24] est proportionnelle à la vitesse de rotation $\dot{\theta}$, on a :

$$
\Delta \Omega=\Omega_{3}-\Omega_{4}=2 \pi S \dot{\theta}
$$

Les deux ondes $\varepsilon_{3}$ et $\varepsilon_{4}$ de fréquence $\omega_{3}$ et $\omega_{4}$ sont générées par le processus de mélange à quatre ondes. La conservation de l'énergie au cours de ce processus impose que l'on ait :

$$
\omega_{3}+\omega_{4}=2 \omega .
$$

Les trois fréquences $\omega, \omega_{3}$ et $\omega_{4}$ étant supposées très proches on peut supposer que la susceptibilité linéaire $\chi_{0}$ et par conséquent l'indice linéaire $n_{0}\left(n_{0}=1+\frac{\chi_{0}}{2}\right)$ dû au milieu atomique remplissant la cavité est le même pour les quatre ondes $\varepsilon_{1}, \varepsilon_{2}, \varepsilon_{3}$ et $\varepsilon_{4}$.

On redéfinit les champs pompes et les champs intra-cavité en tenant compte de cet indice linéaire, on pose donc:

$$
E_{\mathrm{p}}(\mathbf{r}, t)=\sum_{j=1}^{2} \mathcal{R e} \exp -i\left(\omega_{\mathrm{j}} t-\mathbf{k}_{\mathrm{j}} \cdot \mathbf{r}\right)
$$

avec $\omega_{1}=\omega_{2}=\omega$

$$
\begin{gathered}
\left|\mathbf{k}_{1}\right|=\left|\mathbf{k}_{2}\right|=\frac{\omega}{c} n_{0}=\frac{\omega}{c}\left(1+\frac{\chi_{0}}{2}\right) \\
E_{\mathrm{c}}(\mathbf{r}, t)=\sum_{j=3}^{4} \mathcal{R e} \varepsilon_{\mathrm{j}} \exp -i\left(\omega_{\mathrm{j}} t+(-1)^{\mathrm{j}} n_{0} k_{\mathrm{j}} z\right) .
\end{gathered}
$$

Dans ce paragraphe on va étudier l'effet des collisions. Ces collisions ont pour principal effet d'apporter le défaut d'énergie nécessaire pour rendre réelle l'excitation atomique [25]. Dans ce cas on a montré que l'on ne peut plus négliger le rôle de l'effet Doppler pour calculer la susceptibilité non linéaire lorsque les ondes lumineuses forment des réseaux dans le milieu atomique [26]. Pour tenir compte de l'effet Doppler, il faut calculer la contribution à la polarisation d'une classe de vitesse $\mathbf{v}$ puis sommer sur la distribution des vitesses. 
B) Contribution d'Une ClAsse de vitesse. - Pour calculer la contribution d'un atome de vitesse $\mathbf{v}$ à la polarisation non linéaire, il faut résoudre l'équation d'évolution de la matrice densité $\rho(\mathbf{r}, \mathbf{v}, t)$. Cette équation s'écrit :

$$
\left[\left\{\frac{\partial}{\partial t}+\mathbf{v} \cdot \frac{\partial}{\partial \mathbf{r}}\right\} \rho(\mathbf{r}, \mathbf{v}, t)=\frac{\mathrm{d}}{\mathrm{d} t} \rho\right] \mathrm{rel}+\frac{1}{i \hbar}\left[H_{0}, \rho\right]+\frac{1}{i \hbar}[V, \rho] .
$$

$H_{0}$ est l'hamiltonien atomique, $V$ décrit le couplage entre un atome et les ondes incidentes et $\left.\frac{\mathrm{d} \rho}{\mathrm{d} t}\right|_{\text {relax }}$ décrit l'effet de la relaxation (émission spontanée + collisions déphasantes).

Comme les ondes incidentes sont peu saturantes on résout perturbativement cette équation. La solution à l'ordre (3) pour la cohérence optique $\rho_{\text {ba }}$ est égale à [27] :

$$
\begin{gathered}
\rho_{\mathrm{ba}}^{(3)}(\mathbf{r}, \mathbf{v}, t)=\frac{i}{2} \frac{N d}{\hbar} \sum_{\mu} E_{\mu} \frac{\exp -i\left[\omega_{\mu} t-\mathbf{k}_{\mu} \cdot \mathbf{r}+\varphi_{\mu}\right]}{\gamma_{\mathrm{ab}}+i\left(\omega_{0}-\tilde{\omega}_{\mu}\right)} \\
-\frac{i N}{4 \hbar^{3}} \sum_{\mu, \nu, \lambda} \frac{d^{3} E_{\mu} E_{\nu} E_{\lambda}}{\gamma_{\mathrm{ab}}+i\left(\omega_{0}+\tilde{\omega}_{\mu}-\tilde{\omega}_{\nu}-\tilde{\omega}_{\lambda}\right)} \times \\
\times \frac{1}{\gamma_{\mathrm{b}}+i\left(\tilde{\omega}_{\mu}-\tilde{\omega}_{\nu}\right)}\left[\frac{1}{\gamma_{\mathrm{ab}}+i\left(\omega_{0}-\tilde{\omega}_{\nu}\right)}+\frac{1}{\gamma_{\mathrm{ab}}+i\left(\tilde{\omega}_{\mu}-\omega_{0}\right)}\right] \\
\exp -i\left(\psi_{\mu}-\psi_{\nu}-\psi_{\lambda}\right) \exp i\left(\mathbf{k}_{\mu}-\mathbf{k}_{\nu}-\mathbf{k}_{\lambda}\right) \cdot \mathbf{r}
\end{gathered}
$$

avec

$$
\psi_{\mu}=\omega_{\mu} t+\varphi_{\mu}
$$

Dans cette formule les fréquences $\tilde{\omega}_{\mu}$ sont les fréquences des ondes $\boldsymbol{\varepsilon}_{\mu}$ vues par un atome de vitesse $\mathbf{v}$ dans son référentiel. Elles sont donc égales à :

$$
\tilde{\omega}_{\mu}=\omega_{\mu}-\mathbf{k}_{\mu} \cdot \mathbf{v}
$$

Dans les termes d'ordre 3 en champ il faut distinguer les termes d'indice non linéaire des termes de gain dus au processus de mélange à quatre ondes.

C) TERMES D'INDICE NON LINÉAIRE. - On s'intéresse d'abord à la cohérence optique évoluant comme l'onde pompe 1 et proportionnelle à $\varepsilon_{1}$, cette cohérence est égale à :

$$
\begin{aligned}
\frac{i}{2 \hbar} \frac{N d E_{1}}{\gamma_{\mathrm{ab}}+i \delta} \mathrm{e}^{-i\left(\omega_{1} t-\mathbf{k}_{1} \cdot \mathbf{r}+\varphi_{1}\right)} \times & \\
& \times\left\{1-\frac{1}{2} \frac{d^{2} I_{1}}{\hbar^{2} \delta^{2}} \frac{2 \gamma_{\mathrm{ab}}}{\gamma_{\mathrm{b}}}-\frac{1}{2} \sum_{j \neq 1} \frac{d^{2} I_{\mathrm{j}}}{\hbar^{2} \delta^{2}}\left(2 \frac{\gamma_{\mathrm{ab}}}{\gamma_{\mathrm{b}}}+\frac{2 \gamma_{\mathrm{ab}}+i\left(\tilde{\omega}_{\mathrm{j}}-\tilde{\omega}_{1}\right)}{\gamma_{\mathrm{b}}+i\left(\tilde{\omega}_{\mathrm{j}}-\tilde{\omega}_{1}\right)}\right)\right\} .
\end{aligned}
$$

Il faut d'abord remarquer qu'en l'absence de collisions la modification de la cohérence optique par les autres ondes est la même quelle que soit l'onde, est indépendante de $\mathbf{v}$ et vaut le double de la modification de la cohérence optique par l'onde 1. En effet, en l'absence de collisions, on a $2 \gamma_{\mathrm{ab}}=\gamma_{\mathrm{b}}$ ce qui implique que :

$$
\frac{2 \gamma_{\mathrm{ab}}+i\left(\tilde{\omega}_{\mathrm{j}}-\tilde{\omega}_{1}\right)}{\gamma_{\mathrm{b}}+i\left(\tilde{\omega}_{\mathrm{j}}-\tilde{\omega}_{1}\right)}=1 \quad \forall j
$$


Dans ces conditions la cohérence optique $\rho_{\mathrm{ba}}^{(3)}$ est égale à :

$$
\frac{i}{2 \hbar} \frac{N d E_{1}}{\gamma_{\mathrm{ab}}+i \delta} \mathrm{e}^{-i\left(\omega_{1} t-\mathbf{k}_{1} \cdot \mathbf{r}+\varphi_{1}\right)}\left[1-\frac{d^{2}}{2 \hbar^{2} \delta^{2}}\left(I_{1}+2 I_{2}+2 I_{3}+2 I_{4}\right)\right] .
$$

En présence de collisions la modification par l'onde $\varepsilon_{\mathrm{j}}$ va dépendre de $\left(\tilde{\omega}_{\mathrm{j}}-\tilde{\omega}_{1}\right)$ donc de $\mathbf{v}$. En effet on a :

$$
\gamma_{\mathrm{ab}}=\frac{\gamma_{\mathrm{b}}}{2}+\beta p .
$$

La modification de la cohérence optique par une quelconque des ondes $\varepsilon_{j}(j \neq 1)$ s'écrit alors :

$$
-\frac{1}{2} \frac{d^{2} I_{\mathrm{j}}}{\hbar^{2} \delta^{2}}\left[1+\frac{2 \beta p}{\gamma_{\mathrm{b}}}+1+\frac{2 \beta p}{\gamma_{\mathrm{b}}+i\left(\tilde{\omega}_{\mathrm{j}}-\tilde{\omega}_{1}\right)}\right] .
$$

Les deux nouveaux termes proportionnels à $\beta p$ traduisent la création par collision de réseaux réels de population [26]. Ce réseau est statique pour le premier terme, modulé à la fréquence $\tilde{\omega}_{\mathrm{j}}-\tilde{\omega}_{1}$ pour le second. Ces réseaux ont une durée de vie $\tau_{\mathrm{b}}=\frac{1}{\gamma_{\mathrm{b}}}$.

On voit aussi sur la formule (21) que la contribution du réseau modulé dépend en général de la vitesse car $\tilde{\omega}_{j}-\tilde{\omega}_{1}(j \neq 1)$ en dépend. Il faut distinguer le réseau créé par $\varepsilon_{1}$ et $\varepsilon_{3}$ qui est un réseau de grand pas $\left(\frac{\lambda}{\theta}\right)$ des réseaux crées par $\varepsilon_{1}$ et $\varepsilon_{2}$ d'une part et $\varepsilon_{1}$ et $\varepsilon_{4}$ d'autre part qui sont des réseaux de petit pas $\left(\frac{\lambda}{2}\right)$.

Pour calculer la contribution du réseau de grand pas nous supposons que $k \theta u \ll \gamma_{\mathrm{b}} \ll k u$.

Pour les contributions dues aux réseaux de petit pas on a :

$$
\tilde{\omega}_{2}-\tilde{\omega}_{1} \simeq \tilde{\omega}_{4}-\tilde{\omega}_{1}=2 k v_{z} .
$$

Dans ce cas la sommation sur les vitesses fait apparaître un facteur de l'ordre de $\frac{\gamma_{\mathrm{b}}}{k u}$ très petit devant l'unité. On peut dans ces conditions négliger la contribution des réseaux de petit pas. La sommation sur les vitesses donne la contribution des termes d'indice non linéaire à la polarisation $P_{1}(\mathrm{r}, t)$, cette contribution ${ }^{\mathrm{NL}} P_{1}(\mathrm{r}, t)$ est égale à :

$$
{ }^{\mathrm{NL}} P_{1}(\mathbf{r}, t)=\frac{1}{2} \varepsilon_{0} \chi_{1} \varepsilon_{1} \exp -i\left(\omega_{1} t-\mathbf{k}_{1}, \mathbf{r}\right)+\text { c.c. }
$$

On voit que $\chi_{1}$ est égal à :

$$
\chi_{1}=\chi_{0}+\chi^{(3)}\left[2 \frac{\gamma_{\mathrm{ab}}}{\gamma_{\mathrm{b}}} I_{\mathrm{t}}+\left(I_{\mathrm{t}}-I_{1}\right)+2 I_{3} \frac{\beta p}{\gamma_{\mathrm{b}}+i\left(\omega_{3}-\omega_{1}\right)}\right]
$$

avec

$$
\chi_{0}=\frac{N d^{2}}{\varepsilon_{0} \hbar \delta} \quad \text { et } \quad \chi^{(3)}=-\frac{1}{2} \frac{d^{2}}{\hbar^{2} \delta^{2}} \chi_{0} .
$$

Il ne faut pas oublier que $\chi_{1}$ est une fonction de $z$ par suite de la dépendance spatiale de $I_{1}$ et de $I_{2}$ (déplétion des pompes). Un calcul tout à fait analogue permet d'obtenir $\chi_{2}$ :

$$
\chi_{2}=\chi_{0}+\chi^{(3)}\left[2 \frac{\gamma_{\mathrm{ab}}}{\gamma_{\mathrm{b}}} I_{\mathrm{t}}+\left(I_{\mathrm{t}}-I_{2}\right)+2 I_{4} \frac{\beta p}{\gamma_{\mathrm{b}}+i\left(\omega_{4}-\omega_{2}\right)}\right] .
$$


La susceptibilité $\chi_{3}$ (resp. $\chi_{4}$ ) s'obtient à partir de l'expression donnant $\chi_{1}$ (resp. $\chi_{2}$ ) par échange des indices 1 et 3 (resp. les indices 2 et 4).

Dans l'expression de $\chi_{1}$ le dernier terme dû au réseau de grand pas créé par les ondes $\varepsilon_{1}$ et $\delta_{3}$ est responsable d'un échange d'énergie entre ces deux ondes. Cet effet de couplage est appelé mélange à deux ondes et a été observé d'abord dans les photoréfractifs [9] puis dans la vapeur de sodium où il est induit par collision [28]. Cet effet de couplage varie comme une fonction de dispersion en fonction de $\delta \omega$ la différence $\omega_{3}-\omega_{1}$. Le gain par mélange à̀ deux ondes peut être supérieur à l'absorption linéaire ce qui conduit à l'amplification d'une des deux ondes.

Dans notre situation $\left(\delta \omega \ll \gamma_{\mathrm{b}}\right)$ le gain associé à ce processus est égal à $\left|r_{\mathrm{c}}\right| \frac{\beta p|\delta \omega|}{\gamma_{\mathrm{b}}^{2}}$ où $r_{\mathrm{c}}$ est la réflectivité par conjugaison de phase. On supposera que le produit $\beta p|\delta \omega|$ est très petit devant $\gamma_{b}^{2}$ de telle sorte que l'on négligera dans toute la suite le processus de mélange à deux ondes.

En présence de collisions un autre effet apparaît lorsque les pompes sont d'intensités différentes $\left(I_{1} \neq I_{2}\right)$. En effet dans ce cas les susceptibilités $\chi_{3}$ et $\chi_{4}$ sont différentes et leur différence est proportionnelle à $\left(I_{1}-I_{2}\right)$, on a :

$$
\chi_{3}-\chi_{4}=2 \chi^{(3)} \frac{\beta p}{\gamma_{\mathrm{b}}}\left(I_{1}-I_{2}\right) .
$$

Dans ces conditions les fréquences propres de la cavité dépendent du sens de parcours et leur différence est proportionnelle à $\left(I_{1}-I_{2}\right)$, on a donc créé un biais optique [29].

D) TERmes de CONJUgaison de PHASE. - A partir de l'expression (15) on peut calculer pour une classe de vitesse $\mathbf{v}$ la cohérence optique responsable de l'émission d'une onde $\varepsilon_{1}$ par mélange à quatre ondes. En supposant comme précédemment que $k \theta u \ll \gamma_{\mathrm{b}} \ll k u$ on obtient après sommation sur les vitesses la contribution du processus de conjugaison de phase à la polarisation $P_{1}(\mathbf{r}, t)$. Cette contribution ${ }^{C P} P_{1}(\mathbf{r}, t)$ est égale à :

$$
\begin{aligned}
{ }^{\mathrm{CP}} P_{1}(\mathbf{r}, t)=-\frac{i}{2 \hbar^{3}} \frac{N d^{4}}{\left(\gamma_{\mathrm{ab}}+i \delta\right)} & \frac{E_{2} E_{3} E_{4}}{\delta^{2}} \exp -i\left(\omega_{1} t-\mathbf{k}_{1} \cdot \mathbf{r}\right) \times \\
& \times \exp \cdot i\left(\varphi_{2}-\varphi_{3}-\varphi_{4}\right)\left[1+\frac{\beta p}{\gamma_{\mathrm{b}}+i\left(\omega_{2}-\omega_{4}\right)}\right]+\text { c.c. }
\end{aligned}
$$

On définit le coefficient de couplage $K_{12}$ entre $\varepsilon_{1}$ et $\varepsilon_{2}^{*}$ par la formule suivante :

$$
{ }^{\mathrm{CP}} P_{1}(\mathbf{r}, t)=\frac{1}{2} \varepsilon_{0} K_{12} \varepsilon_{2}^{*} \exp -i\left(\omega_{1} t-\mathbf{k}_{1} \cdot \mathbf{r}\right)+\text { c.c. }
$$

On voit que $K_{12}$ est égal à :

$$
K_{12}=2 \chi^{(3)} \varepsilon_{3} \varepsilon_{4}\left[1+\frac{\beta p}{\gamma_{\mathrm{b}}+i\left(\omega_{2}-\omega_{4}\right)}\right]=K \varepsilon_{3} \varepsilon_{4}
$$

Comme les ondes $\varepsilon_{3}$ et $\varepsilon_{4}$ sont conjuguées l'une de l'autre on a :

$$
\omega_{3}+\omega_{4}=\omega_{1}+\omega_{2}=2 \omega .
$$

Il est alors facile de montrer que :

$$
K_{21}=K^{*} \varepsilon_{3} \varepsilon_{4}, \quad K_{43}=K \varepsilon_{1} \varepsilon_{2} \quad \text { et } \quad K_{34}=K^{*} \varepsilon_{1} \varepsilon_{2} .
$$


L'approximation consistant à négliger le gain par mélange à deux ondes devant celui par mélange à quatre ondes $\left(\beta p|\delta \omega| \ll \gamma_{\mathrm{b}}^{2}\right)$ permet d'approximer le coefficient $K$ par sa partie réelle $K_{\mathrm{r}}$ :

$$
K \simeq K_{\mathrm{r}}=2 \chi^{(3)}\left[1+\frac{\beta p}{\gamma_{\mathrm{b}}}\right]
$$

E) EXPRESSION DES COMPOSANTES DE FOURIER DE LA POLARISATION. - La polarisation à l'ordre 3 est obtenue en sommant les contributions dues aux termes d'indice non linéaire à celles dues au processus de mélange à quatre ondes. Connaissant la polarisation, on calcule aisément les composantes de Fourier $\mathfrak{T}_{\mathrm{j}}$, on trouve :

$$
\mathfrak{T}_{1,2}(z)=\varepsilon_{0} \chi_{1,2}(z) \varepsilon_{1,2}(z)+\varepsilon_{0} K \varepsilon_{3} \varepsilon_{4} \varepsilon_{2,1}^{*}(z)
$$

De même on trouve que les composantes $\mathfrak{T}_{3}$ et $\mathfrak{T}_{4}$ sont égales à :

$$
\begin{gathered}
\mathfrak{T}_{3,4}=\varepsilon_{0} \bar{\chi}_{3,4} \varepsilon_{3,4}+\varepsilon_{0} K{\overline{\varepsilon_{1}}}_{2} \varepsilon_{4,3}^{*} \\
\bar{\chi}_{3,4}=\frac{1}{L} \int_{0}^{L} \chi_{3,4}(z) \mathrm{d} z \\
\overline{\boldsymbol{\varepsilon}}_{1} \varepsilon_{2}=\frac{1}{L} \int_{0}^{L} \varepsilon_{1} \varepsilon_{2} \mathrm{~d} z .
\end{gathered}
$$

F) CAlcul Du Gain SATURÉ. - Dans ce paragraphe, nous nous limitons au cas où la déplétion des ondes pompes est le processus dominant de saturation. On a montré précédemment que c'est le cas lorsque l'épaisseur optique $k \chi_{0} L$ est très grande devant l'unité [18]. Pour calculer la déplétion des ondes pompes il faut calculer leur évolution au cours de la traversée de la cavité en anneau de longueur $L$.

Il faut donc résoudre les équations d'évolution couplées (9.c) et (9.d) qui compte tenu du choix particulier des modules des vecteurs d'onde $\mathbf{k}_{1}$ et $\mathbf{k}_{2}$, s'écrivent :

$$
\begin{aligned}
& -2 i k \frac{\partial \mathcal{E}_{1}}{\partial z}-2 i \frac{\omega}{c^{2}} \frac{\partial \mathcal{E}_{1}}{\partial t}=+\mu_{0} \omega^{2}\left(\mathfrak{T}_{1}-\varepsilon_{0} \chi_{0} \varepsilon_{1}\right) \\
& +2 i k \frac{\partial \mathcal{E}_{2}}{\mathrm{~d} z}-\frac{2 i \omega}{c^{2}} \frac{\partial \mathcal{E}_{2}}{\partial t}=\mu_{0} \omega^{2}\left(\mathfrak{T}_{2}-\varepsilon_{0} \chi_{0} \varepsilon_{2}\right) .
\end{aligned}
$$

Comme l'évolution temporelle des champs pompes provient uniquement de celle des ondes intra-cavité et que leur temps caractéristique d'évolution est très long devant le temps de traversée de la cellule on peut considérer que les champs pompes suivent adiabatiquement les champs intra-cavité. Dans ce cas l'équation d'évolution du champ pompe $\mathcal{E}_{1}(z, t)$ s'écrit :

$$
\frac{\partial \varepsilon_{1}}{\partial z}=+\frac{i k}{2 \varepsilon_{0}}\left(\mathfrak{T}_{1}-\varepsilon_{0} \chi_{0} \varepsilon_{1}\right)
$$

avec

$$
\left(\mathfrak{T}_{1}-\varepsilon_{0} \chi_{0} \varepsilon_{1}\right)=\varepsilon_{0}\left(\chi_{1}-\chi_{0}\right) \varepsilon_{1}+\varepsilon_{0} K \varepsilon_{3} \varepsilon_{4} \varepsilon_{2}^{*}
$$

On suppose que les modifications spatiales de $\varepsilon_{1}$ induites par la polarisation non linéaire sont très faibles. Par conséquent on traite leur effet de manière perturbative et on se limite à 
l'ordre 1 en $k \chi^{(3)} I L$. Si on note $\varepsilon_{1}(0)$ et $\varepsilon_{2}(L)$ les champs pompes entrant dans la cellule et $\varepsilon_{1}(L), \varepsilon_{2}(0)$ les champs pompes sortant on obtient :

$$
\begin{aligned}
& \varepsilon_{1}(L)=\varepsilon_{1}(0)+i \frac{k}{2} L\left[\chi_{1}^{\mathrm{NL}} \varepsilon_{1}(0)+K \varepsilon_{3} \varepsilon_{4} \varepsilon_{2}^{*}(L)\right] \\
& \varepsilon_{2}(0)=\varepsilon_{2}(L)+i \frac{k}{2} L\left[\chi_{2}^{\mathrm{NL}} \varepsilon_{2}(L)+K \varepsilon_{3} \varepsilon_{4} \varepsilon_{1}^{*}(0)\right]
\end{aligned}
$$

avec

$$
\begin{aligned}
& \chi_{1}^{\mathrm{NL}}=\chi_{1}-\chi_{0}=\chi^{(3)}\left[2 I_{\mathrm{t}}-I_{1}+\frac{2 \beta p}{\gamma_{\mathrm{b}}}\left(I_{\mathrm{t}}+I_{3}\right)\right] \\
& \chi_{2}^{\mathrm{NL}}=\chi_{2}-\chi_{0}=\chi^{(3)}\left[2 I_{\mathrm{t}}-I_{2}+\frac{2 \beta p}{\gamma_{\mathrm{b}}}\left(I_{\mathrm{t}}+I_{4}\right)\right] .
\end{aligned}
$$

Nous avons montré au paragraphe 3-C qu'il est intéressant d'étudier la situation où les intensités $I_{1}$ et $I_{2}$ des ondes pompes sont différentes. Pour simplifier les calculs on limitera notre étude au cas où la différence $\left(I_{1}-I_{2}\right)$ est très petite devant $I_{0}$ la valeur moyenne des intensités pompes $\left(I_{1}+I_{2}=2 I_{0}\right)$. Dans ces conditions on pourra en première approximation remplacer $\chi_{1}^{\mathrm{NL}}$ et $\chi_{2}^{\mathrm{NL}}$ par leurs valeurs pour $I_{1}=I_{2}=I_{0}$ :

$$
\chi_{1}^{\mathrm{NL}}=\chi_{2}^{\mathrm{NL}}=\chi^{\mathrm{NL}}=\chi^{(3)}\left[3 I_{0}+4 I+\frac{2 \beta p}{\gamma_{\mathrm{b}}}\left(2 I_{0}+3 I\right)\right]
$$

où $I$ est la valeur de l'intensité des ondes intra-cavité $\left(I_{3}=I_{4}=I\right)$. Avec ces hypothèses on trouve que la valeur moyenne spatiale du produit $\varepsilon_{1} \varepsilon_{2}$ est égale à :

$$
{\overline{\mathcal{E}_{1}}}_{\varepsilon_{2}}=I_{0} \exp -i \Phi_{\mathrm{p}}^{\prime}+i \alpha^{\prime} I_{0} \varepsilon_{3} \varepsilon_{4}
$$

avec $\alpha^{\prime}=\frac{1}{2} k K L$

$$
\Phi_{\mathrm{p}}^{\prime}=\varphi_{1}+\varphi_{2}-\frac{k}{2} \chi^{\mathrm{NL}} L=\Phi_{\mathrm{p}}-\frac{k}{2} \chi^{\mathrm{NL}} L
$$

$\operatorname{avec} \Phi_{\mathrm{p}}=\varphi_{1}+\varphi_{2}$

On montre de la même manière que les valeurs moyennes spatiales des intensités $I_{1}$ et $I_{2}$ sont égales à :

$$
\bar{I}_{\mathrm{i}}=I_{\mathrm{i}}+\alpha^{\prime} I_{0} E_{3} E_{4} \sin \left(\Phi_{\mathrm{c}}-\Phi_{\mathrm{p}}\right) \quad(i=1,2)
$$

$\operatorname{avec} \Phi_{\mathrm{c}}=\varphi_{3}+\varphi_{4}$

G) EQuATIONS DE LAMB.

a) Equation d'évolution. - Connaissant les composañtes de Fourier $\mathfrak{T}_{3}$ et $\mathfrak{T}_{4}$ on en déduit les équations d'évolution des $E_{\mathrm{j}}$ et des $\varphi_{\mathrm{j}}(j=3,4)$. L'équation d'évolution de $E_{3}$ s'écrit :

$$
\dot{E}_{3}+\frac{\sigma}{2 \varepsilon_{0}} E_{3}=-\frac{\omega_{3}}{2} K I_{0} E_{4}\left[\sin \left(\Phi_{\mathrm{c}}-\Phi_{\mathrm{p}}^{\prime}\right)+\alpha^{\prime} \sqrt{I_{3} I_{4}}\right]
$$

On montre de la même façon que l'équation d'évolution de $\varphi_{3}$ s'écrit :

$$
\omega_{3}+\dot{\varphi}_{3}-\Omega_{3}=-\frac{\omega_{3}}{2}\left\{\chi^{(3)}\left(\overline{2 I}_{\mathrm{t}}-I_{3}\right)+2 \frac{\beta p}{\gamma_{\mathrm{b}}} \chi^{(3)}\left(\bar{I}_{\mathrm{t}}+\bar{I}_{1}\right)+K I_{0} \sqrt{\frac{I_{4}}{I_{3}}} \cos \left(\Phi_{\mathrm{c}}-\Phi_{\mathrm{p}}^{\prime}\right)\right\} .
$$


L'équation (41a) et celle qui s'en déduit par échange des indices 3 et 4 montrent que les intensités sont égales en régime stationnaire. On en déduit alors que la différence entre les fréquences $\omega_{3}$ et $\omega_{4}$ est égale à :

$$
\begin{aligned}
\omega_{3}-\omega_{4} & =\Omega_{3}-\Omega_{4}-\omega \frac{\beta p}{\gamma_{\mathrm{b}}} \chi^{(3)}\left(I_{1}-I_{2}\right) \\
& =2 \pi S \dot{\theta}-\omega \frac{\beta p}{\gamma_{\mathrm{b}}} \chi^{(3)}\left(I_{1}-I_{2}\right)
\end{aligned}
$$

Cette équation nous montre clairement qu'un oscillateur en anneau à conjugaison de phase peut servir de gyromètre optique et que son facteur d'échelle est le même que celui d'un gyrolaser traditionnel de mêmes dimensions. De plus cette équation montre qu'en présence de collisions et pour une valeur suffisante de $\left(I_{1}-I_{2}\right)$, un biais optique permettant de sortir de la zone aveugle peut être obtenu. En effet, la rétrodiffusion sur les miroirs couplant les deux ondes tournant en sens opposé dans l'anneau, un verrouillage de leur fréquence se produit lorsque la vitesse de rotation est en valeur absolue inférieure à $\dot{\theta}_{\mathrm{c}}$. Dans un gyrolaser traditionnel le problème peut être résolu en ajoutant un mouvement mécanique supplémentaire de rotation, ce qui permet de sortir de la zone aveugle et de mesurer des vitesses de rotation inférieures à $\dot{\theta}_{\mathrm{c}}$. On voit que dans notre cas, ceci peut être réalisé optiquement en modulant la différence $\left(I_{1}-I_{2}\right)$. En particulier le fait de pouvoir moduler en créneau carré cette quantité pourrait permettre d'augmenter la précision de la mesure de $\dot{\theta}$ par rapport à une activation mécanique qui est forcément sinusoïdale [23, 24].

b) Intensités et phases en régime stationnaire. - Il apparaît clairement à partir de l'équation (41a) et de celle obtenue par échange des indices 3 et 4 que ces équations admettent une solution stationnaire pour laquelle $E_{3}=E_{4}$. En appelant $I$ l'intensité de ces ondes $I=E_{3}^{2}=E_{4}^{2}$, nous trouvons :

$$
g^{\prime} \sin \left(\Phi_{\mathrm{c}}-\Phi_{\mathrm{p}}^{\prime}\right)-\gamma=s I
$$

De même l'équation (41b) et celle obtenue par échange des indices 3 et 4 donnent :

$$
\Delta=\omega_{4}-\Omega_{4}^{\prime}=\omega_{3}-\Omega_{3}^{\prime}=g^{\prime} \cos \left(\dot{\Phi}_{\mathrm{c}}-\Phi_{\mathrm{p}}^{\prime}\right)+\frac{3}{2} g \frac{I}{I_{0}}+2 g x \frac{I}{I_{0}}
$$

avec

$$
\begin{gathered}
g^{\prime}=-\omega \chi{ }^{(3)} I_{0}\left(1+\frac{\beta p}{\gamma_{\mathrm{b}}}\right)=g(1+x) \\
s=-g^{\prime} \alpha^{\prime} \\
\gamma=\frac{\sigma}{2 \varepsilon_{0}}
\end{gathered}
$$

$\Omega_{3,4}^{\prime}$ sont les fréquences de la cavité modifiées par la présence du milieu atomique, elles sont d'après la formule $(41 \mathrm{~b})$ égales à :

$$
\Omega_{3,4}^{\prime}=\Omega\left(1 \pm \frac{\pi S \dot{\theta}}{\Omega}+\frac{\chi_{0}}{2}+K I_{0}+\frac{\beta p}{\gamma_{\mathrm{b}}} \chi^{(3)} I_{1,2}\right)^{-1}
$$

où $\Omega$ est la fréquence propre de la cavité vide et immobile. 
A partir du système précédent (formules 43) on obtient aisément la valeur de $g_{\mathrm{s}}^{\prime}$ le gain au seuil, en faisant $I=0$ dans les deux équations :

$$
g_{\mathrm{s}}^{\prime}=\frac{c}{L}\left|r_{\mathrm{c}}\right|=\sqrt{\gamma^{2}+\Delta^{2}} \text {. }
$$

Dans cette expression $r_{\mathrm{c}}=\sqrt{R_{\mathrm{c}}}$ où $R_{\mathrm{c}}$ est le coefficient de réflexion du miroir à conjugaison de phase pour une onde progressive [18]. On peut réexprimer cette condition de seuil en fonction de $\eta$ le déphasage sur un tour $\left(\Delta=\frac{c}{L} \eta\right)$ et de la transmission $T$ du miroir de sortie de la cavité $\left(\gamma=\frac{c}{L} \frac{T}{2}\right)$. On voit que la condition de seuil en fonction de $\eta$ et de $T$ s'écrit :

$$
R_{\mathrm{c}}=\eta^{2}+\frac{T^{2}}{4}
$$

Cette condition coïncide avec celle trouvée par l'approche spatiale [18]: le seuil d'apparition d'une oscillation est très bas et varie comme le carré des pertes pour une cavité accordée. Ce seuil très bas montre qu'une onde stationnaire de phase $\Phi_{c}$ appropriée peut extraire beaucoup plus d'énergie des ondes pompes qu'une onde progressive. Finalement il faut remarquer que la valeur de $\Phi_{\mathrm{c}}$ est bloquée sur $\Phi_{\mathrm{p}}$ la somme des phases $\varphi_{1}$ et $\varphi_{2}$ des deux ondes pompes.

Etudions maintenant les valeurs de $I$ et de $\Phi_{\mathrm{c}}$ légèrement au-dessus du seuil. Notre étude étant limitée au fonctionnement de l'oscillateur très près du seuil, on suppose donc que :

$$
g^{\prime}-\gamma \ll \gamma .
$$

Dans ce cas la phase $\Phi_{\mathrm{c}}-\Phi_{\mathrm{p}}^{\prime}$ va se bloquer à une valeur très proche de $+\frac{\pi}{2}$ (si $\left.\chi_{0}>0\right)$. En posant $\Phi_{\mathrm{c}}-\Phi_{\mathrm{p}}^{\prime}=\frac{\pi}{2}+\Delta \Phi$ le système d'équations se réécrit :

$$
\left\{\begin{array}{l}
g^{\prime}\left(1-\frac{\Delta \dot{\Phi}^{2}}{2}\right)-\gamma=s I \\
\Delta+g^{\prime} \Delta \Phi=\frac{3}{2} g\left(1+\frac{4}{3} x\right) \frac{I}{I_{0}} .
\end{array}\right.
$$

Ce système nous montre que l'intensité $I$ est l'ordonnée du point d'intersection entre une droite et une parabole (Fig. 2). Un tel système peut avoir deux solutions non nulles : pour qu'il en soit ainsi il faut que la pente de la droite soit inférieure à la pente de la tangente à la parabole au point A d'abscisse $-\Delta \Phi_{\mathrm{c}}\left(\Delta \Phi_{\mathrm{c}}\right.$ est défini par $\left.g^{\prime}\left(1-\frac{\Delta \Phi_{\mathrm{c}}^{2}}{2}\right)=\gamma\right)$. Comme on suppose que la cavité est une bonne cavité on trouve que cette condition est vérifiée sauf dans une toute petite plage d'intensité pompe juste au-dessus du seuil.

Pour une valeur de $\Delta$ on trouve une, deux ou trois solutions (la solution $I=0$ est toujours possible en dehors de l'intervalle $\left.\left[-\Delta \Phi_{c}, \Delta \Phi_{c}\right]\right)$. On voit sur la figure 3 qu'en fonction du sens de variation de la longueur de la cavité on peut obtenir deux valeurs différentes de I. Ceci est le schéma classique de la bistabilité dispersive [30-32].

c) Effet de la rétrodiffusion. - Dans ce paragraphe on va introduire l'effet de la rétrodiffusion due aux miroirs. La rétrodiffusion totale qui est la somme des rétrodiffusions dues aux différents miroirs de la cavité est caractérisée par une amplitude $r$ et un déphasage $\varepsilon[23,24]$. 


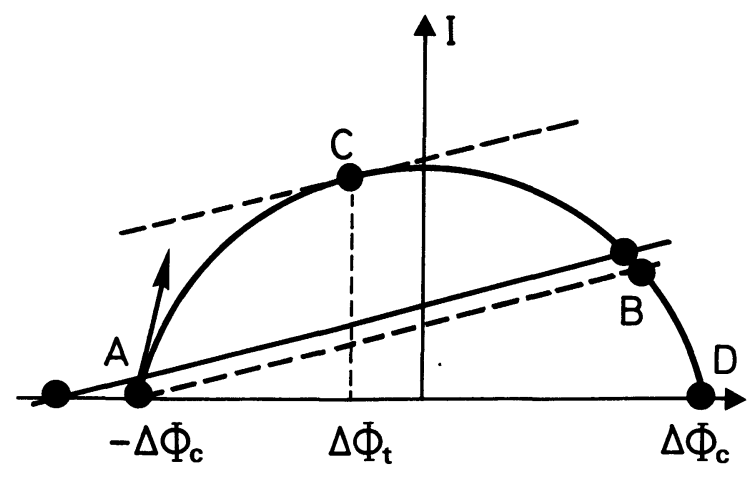

Fig. 2. - Les solutions du système d'équations (43) correspondent à l'intersection d'une droite et d'une parabole. Chaque solution physique est indiquée par un point. Les lignes pointillées correspondent à des situations où un brusque saut d'intensité se produit lorsqu'on varie $L$.

[The solutions of (43) correspond to the intersection of the solid line and the parabola. The possible physical solutions are indicated by dots. The dashed lines correspond to the situations where a sudden jump in intensity is expected when $L$ is scanned.]

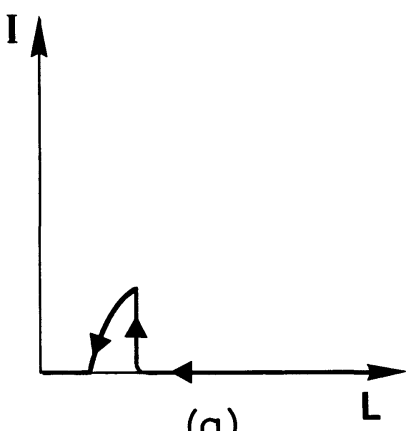

(a)

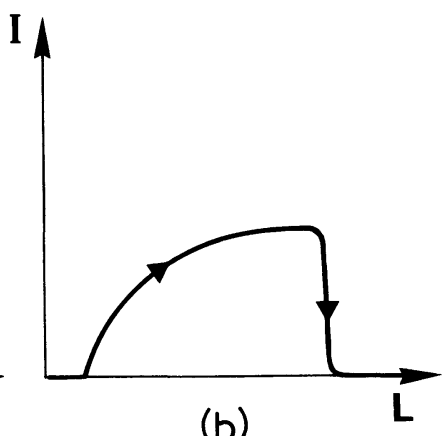

(b)

Fig. 3. - Variation de l'intensité émise $I$ en fonction de la longueur $L$ de la cavité. Quand la longueur de la cavité change, l'abscisse du point d'intersection de la droite et de l'axe horizontal change (voir Fig. 2). Partant de la ligne continue de la figure 2 la solution $I=0$ reste une solution lorsque $L$ décroît jusqu'à ce que l'abscisse coïncide avec $\mathrm{A}$. Pour une valeur de $L$ légèrement inférieure, le seul point stable devient le point $\mathrm{B}$ qui correspond à une intensité $I \neq 0$. Lorsqu'on continue à diminuer $L$ le point de fonctionnement décrit la branche BD de la parabole. Lorsque l'abscisse de la droite est supérieure à l'abscisse du point $\mathrm{D}$ l'intensité émise est nulle. Ceci est représenté sur la figure (3a). Lorsque la longueur $L$ croît (Fig. $3 b$ ) le système suit par continuité la solution non nulle jusqu'à ce que le point $\mathrm{C}$ soit atteint. Ensuite la solution d'intensité non nulle étant instable le système cesse d'osciller.

[Variation of the emitted intensity as a function of the length of the cavity. When the length of the cavity changes, the abscissa of the solid line of figure 2 varies. Starting from the solid line of figure 2 we see that $I=0$ remains a solution when $L$ increases until the abscissa coincides with A. For slightly larger values of $L$, the only stable solution is $\mathrm{B}$, for which $I \neq 0$. This corresponds to the curve shown on (Fig. 3a). When the length decreases (Fig. 3b), the system continuously follows the nonzero solution until the point $\mathrm{C}$ of figure 3 is reached.] 
A chaque tour de cavité la rétrodiffusion rajoute au vecteur $\varepsilon_{3}$ un vecteur qui vaut :

avec

$$
\begin{gathered}
r \mathrm{e}^{i \varepsilon} E_{4} \exp i\left(\psi_{3}-\psi_{4}\right) \\
\psi_{3,4}=\left(\omega_{3,4}\right) t+\varphi_{3,4} .
\end{gathered}
$$

Comme $r$ est très petit devant l'unité on $\mathbf{a}$ :

$$
\begin{aligned}
\frac{\mathrm{d}}{\mathrm{d} t} \psi_{3} & =\frac{c}{L} r \frac{E_{4}}{E_{3}} \sin (\psi+\varepsilon) \\
\frac{\mathrm{d}}{\mathrm{d} t} E_{3} & =\frac{c}{L} r E_{4} \cos (\psi+\varepsilon)
\end{aligned}
$$

avec

$$
\psi=\psi_{3}-\psi_{4} .
$$

On en déduit immédiatement l'équation d'évolution de $\psi$, on a :

$$
\frac{\mathrm{d}}{\mathrm{d} t} \psi=\omega_{\ell}\left[\left(\frac{E_{3}}{E_{4}}+\frac{E_{4}}{E_{3}}\right) \sin \psi \cos \varepsilon-\left(\frac{E_{3}}{E_{4}}-\frac{E_{4}}{E_{3}}\right) \sin \varepsilon \cos \psi\right]
$$

avec

$$
\omega_{\ell}=\frac{c}{L} r
$$

d) Equations globales d'évolution. Zone aveugle active. - Les équations globales d'évolution s'obtiennent en ajoutant l'effet de la rétrodiffusion aux équations d'évolution du $\S 3 \mathrm{Ga}$.

On obtient alors :

$$
\begin{gathered}
\dot{E}_{3}+\gamma E_{3}=g^{\prime} E_{4}\left[\sin \left(\Phi_{\mathrm{c}}-\Phi_{\mathrm{p}}^{\prime}\right)+\alpha^{\prime} E_{3} E_{4}\right]+\omega_{\ell} E_{4} \cos (\psi+\varepsilon) \\
\dot{E}_{4}+\gamma E_{4}=g^{\prime} E_{3}\left[\sin \left(\Phi_{\mathrm{c}}-\Phi_{\mathrm{p}}^{\prime}\right)+\alpha^{\prime} E_{3} E_{4}\right]+\omega_{\ell} E_{3} \cos (\psi-\varepsilon) \\
\dot{\Phi}_{\mathrm{c}}+2 \omega-\left(\Omega_{3}+\Omega_{4}\right)=-\frac{\omega}{2}\left(\chi_{3}+\chi_{4}\right)+g^{\prime} \cos \left(\Phi_{\mathrm{c}}-\Phi_{\mathrm{p}}^{\prime}\right)\left[\frac{E_{3}}{E_{4}}+\frac{E_{4}}{E_{3}}\right]+ \\
+\omega_{\ell}\left(\frac{E_{4}}{E_{3}}-\frac{E_{3}}{E_{4}}\right) \sin \psi \cos \varepsilon+\omega_{\ell}\left(\frac{E_{4}}{E_{3}}+\frac{E_{3}}{E_{4}}\right) \cos \psi \sin \varepsilon \\
\dot{\psi}=2 \pi S \dot{\theta}-\omega \frac{\beta p}{\gamma_{\mathrm{b}}} \chi^{(3)}\left[\bar{I}_{1}-\bar{I}_{2}\right]+\frac{\omega}{2} \chi^{(3)}\left[I_{3}-I_{4}\right]+ \\
+g^{\prime}\left(\frac{E_{4}}{E_{3}}-\frac{E_{3}}{E_{4}}\right) \cos \left(\Phi_{\mathrm{c}}-\Phi_{\mathrm{p}}^{\prime}\right)+\omega_{\ell}\left(\frac{E_{4}}{E_{3}}+\frac{E_{3}}{E_{4}}\right) \sin \psi \cos \varepsilon+\omega_{\ell}\left(\frac{E_{4}}{E_{3}}-\frac{E_{3}}{E_{4}}\right) \cos \psi \sin \varepsilon .
\end{gathered}
$$

On va d'abord résoudre le cas particulier $\varepsilon=0$. En effet, dans ce cas, la rétrodiffusion conserve l'égalité entre $I_{3}$ et $I_{4}$. L'équation d'évolution de la phase s'écrit alors :

$$
\dot{\psi}=\Delta \Omega+2 \omega_{\ell} \sin \psi
$$

Cette équation est l'équation d'Adler [33] que l'on rencontre dans l'étude des gyrolasers traditionnels [23], cette équation admet une solution stationnaire $\psi_{\mathrm{s}}$ tant que $\Delta \Omega / 2 \omega_{\ell}$ est compris entre -1 et 1 . Cette solution est :

$$
\psi_{\mathrm{s}}=-\operatorname{Arcsin} \frac{\Delta \Omega}{2 \omega_{\ell}} .
$$


En l'absence de biais optique $\left(I_{1}=I_{2}\right)$ la différence $\Delta \Omega$ est égale à $(2 \pi S \dot{\theta})$. On voit que la vitesse de rotation critique $\dot{\theta}_{c}$ est égale à :

$$
\dot{\theta}_{\mathrm{c}}=\frac{\omega_{\ell}}{\pi S}=\frac{c r}{L \pi S} .
$$

Si $|\Delta \Omega|$ est supérieur à $2 \omega_{\ell}$ alors il n'existe plus de solution stationnaire. Il existe néanmoins une solution analytique [34] qui est une fonction périodique du temps de période $T=2 \pi \Gamma^{-1}$ avec

$$
\Gamma=\sqrt{\Delta \Omega^{2}-4 \omega_{\ell}^{2}} .
$$

Lorsque $\varepsilon$ est différent de zéro le problème est plus compliqué car les intensités $I_{3}$ et $I_{4}$ sont différentes et leur différence $\Delta I=I_{3}-I_{4}$ est couplée à $\psi$ par la rétrodiffusion. Néanmoins, cette différence étant due à la rétrodiffusion on peut la supposer très petite devant $I$ la valeur moyenne des intensités $\left(I=\frac{I_{3}+I_{4}}{2}\right)$. Dans ces conditions on peut linéariser les équations d'évolution de $I_{3}, I_{4}$ et de $\Psi$. L'équation d'évolution de $\Delta I$ est alors égale à :

$$
\frac{\mathrm{d}}{\mathrm{d} t} \Delta I+2 \gamma \Delta I=-\left(4 \omega_{\ell} \sin \varepsilon \sin \psi\right) I
$$

Lorsque les intensités $I_{3}$ et $I_{4}$ sont différentes, on voit apparaître dans l'équation d'évolution de la phase un nouveau terme proportionnel à $I_{3}-I_{4}$. Son origine physique est la même que celle du biais optique proportionnel à $\left(I_{1}-I_{2}\right)$, néanmoins ce nouveau terme existe même sans collisions contrairement au biais optique. En linéarisant l'équation d'évolution de $\psi$ on obtient :

$$
\dot{\psi}=\Delta \Omega-\frac{g}{2} \cdot \frac{I}{I_{0}} \cdot \frac{\Delta I}{I}+g^{\prime} \frac{\Delta I}{I} \Delta \Phi+2 \omega_{\ell} \sin \psi \cos \varepsilon-\omega_{\ell} \frac{\Delta I}{I} \cos \psi \sin \varepsilon
$$

Pour trouver l'expression de la zone aveugle on se place à une vitesse $\dot{\theta}$, très proche de $\dot{\theta}_{\mathrm{c}}$, dans ces conditions $\psi$ évolue très lentement et on peut supposer que $\Delta I$ suit adiabatiquement, on a donc:

$$
\frac{\Delta I}{I}=-\frac{2 \omega_{\ell}}{\gamma} \sin \varepsilon \sin \psi .
$$

On voit que la condition de validité du traitement est que la rétrodiffusion due aux miroirs soit très faible devant les pertes de la cavité $\left(\omega_{\ell} \ll \gamma\right)$.

Dans ces conditions l'équation d'évolution de $\psi$ s'écrit :

$$
\dot{\psi}=\Delta \Omega+a \sin \psi+b \sin 2 \psi
$$

avec

$$
\begin{aligned}
& a=2 \omega_{\ell} \cos \varepsilon-2\left(g^{\prime} \Delta \Phi-\frac{g}{2} \frac{I}{I_{0}}\right) \frac{\omega_{\ell}}{\gamma} \sin \varepsilon \\
& b=\frac{\omega_{\ell}^{2}}{\gamma} \sin ^{2} \varepsilon .
\end{aligned}
$$


Cette équation est de la forme $\dot{\psi}=\Delta \Omega+f(\psi)$ où $f(\psi)$ est une fonction périodique de $\psi$ qui varie entre $-f_{\max }$ et $f_{\max }$. Si $\Delta \Omega$ (que l'on suppose positif) est inférieur ou égal à $f_{\max }$ alors cette équation admet une solution stationnaire. La fonction $f(\psi)$ étant de la forme $a \sin \psi+b \sin 2 \psi$ on montre aisément que son maximum est égal à $\sqrt{a^{2}+4 b^{2}}$ lorsque $a$ est très grand devant $b$. Cette dernière condition est toujours réalisée sauf lorsque $\varepsilon$ est très voisin de $\frac{\pi}{2}$. On en déduit que la vitesse de rotation critique $\dot{\theta}_{\mathrm{c}}$ est égale à :

$$
\frac{1}{2 \pi S} \sqrt{a^{2}+4 b^{2}} \text {. }
$$

Lorsque $\varepsilon$ est différent de zéro on voit que la zone aveugle dépend du point de fonctionnement. En effet, dans ces conditions le coefficient $a$, qui d'après (58) est égal à :

$$
2 \omega_{\ell} \cos \varepsilon-2 \frac{\omega_{\ell}}{\gamma} \sin \varepsilon\left(g^{\prime} \Delta \Phi-\frac{g}{2} \frac{I}{I_{0}}\right)
$$

dépend du point de fonctionnement par l'intermédiaire de $\Delta \Phi$ et de $I$. On voit en particulier que le coefficient $a$ peut s'annuler, dans ces conditions la zone aveugle est égale à $b$ et est par conséquent très petite.

\section{Etude expérimentale.}

A) Description Du mONTAge. - Pour étudier expérimentalement les propriétés d'un oscillateur utilisant le mélange à quatre ondes nous avons utilisé le montage représenté par la figure 4.

La vapeur atomique de sodium est contenue dans une cellule en quartz à angle de Brewster qui contient aussi 3 torrs d'hélium. La cellule est chauffée à $165^{\circ}$.

Le faisceau de sortie du laser à colorant monomode (largeur du laser $\simeq 1 \mathrm{MHz}$ ), d'intensité $I$, traverse un cristal électro-optique suivi d'un prisme de Glan. Ce dispositif divise le faisceau incident en deux faisceaux $\varepsilon_{1}$ et $\varepsilon_{2}$ polarisés orthogonalement. Les intensités respectives $I_{1}$ et $I_{2}$ de ces deux faisceaux dépendent de la tension appliquée sur le cristal électro-optique, leur somme restant constante et égale à $2 I_{0}$. L'utilisation d'une lame demi-onde permet de faire tourner la polarisation du faisceau $\varepsilon_{1}$ de façon à obtenir deux faisceaux pompes de même polarisation linéaire verticale. La puissance typique d'un des faisceaux pompes à l'entrée de la cellule est de $150 \mathrm{~mW}$ et chacun des faisceaux est focalisé sur une tache dont le diamètre est égal à $300 \mu \mathrm{m}$.

La cavité rectangulaire est constituée de quatre miroirs plans $\mathbf{M}_{1}, \mathbf{M}_{2}, \mathbf{M}_{3}$ et $\mathbf{M}_{4}$ parfaitement réfléchissants et sa longueur $L$ est égale à $136 \mathrm{~cm}\left(\frac{c}{L}=220 \mathrm{MHz}\right)$. En plaçant une lentille de focale $f=200 \mathrm{~mm}$ au milieu du bras $\mathbf{M}_{1} \mathbf{M}_{2}$ et une identique au milieu du bras $\mathbf{M}_{3} \mathbf{M}_{4}$ on impose deux "waists » identiques pour le mode fondamental de la cavité au milieu des bras $\mathrm{M}_{2} \mathrm{M}_{3}$ et $\mathrm{M}_{1} \mathrm{M}_{4}$. Les "waists » ont un diamètre de $170 \mu$. On place la cellule de sodium au milieu du bras $\mathbf{M}_{1} \mathbf{M}_{4}$. Afin d'extraire de l'énergie de la cavité on a placé une lame de verre dans le bras $\mathbf{M}_{2} \mathbf{M}_{3}$. Cette lame est orientée à $45^{\circ}$ et une de ces faces a subi un traitement anti-reflet. A chaque tour cette lame permet d'extraire $15 \%$ de l'énergie des faisceaux circulant dans la cavité. Son rôle est donc identique à celui d'un miroir de coefficient de transmission $T=0,15$. Le miroir $\mathrm{M}_{1}$ est collé sur une cale piézo-électrique, ce qui permet de balayer la longueur de la cavité. Enfin, lorsqu'on veut travailler à longueur de cavité constante, on utilise un faisceau auxiliaire émis par un laser $\mathrm{He}-\mathrm{Ne}$ stabilisé. Ce faisceau qui est polarisé horizontalement est injecté dans la cavité par l'intermédiaire du miroir 


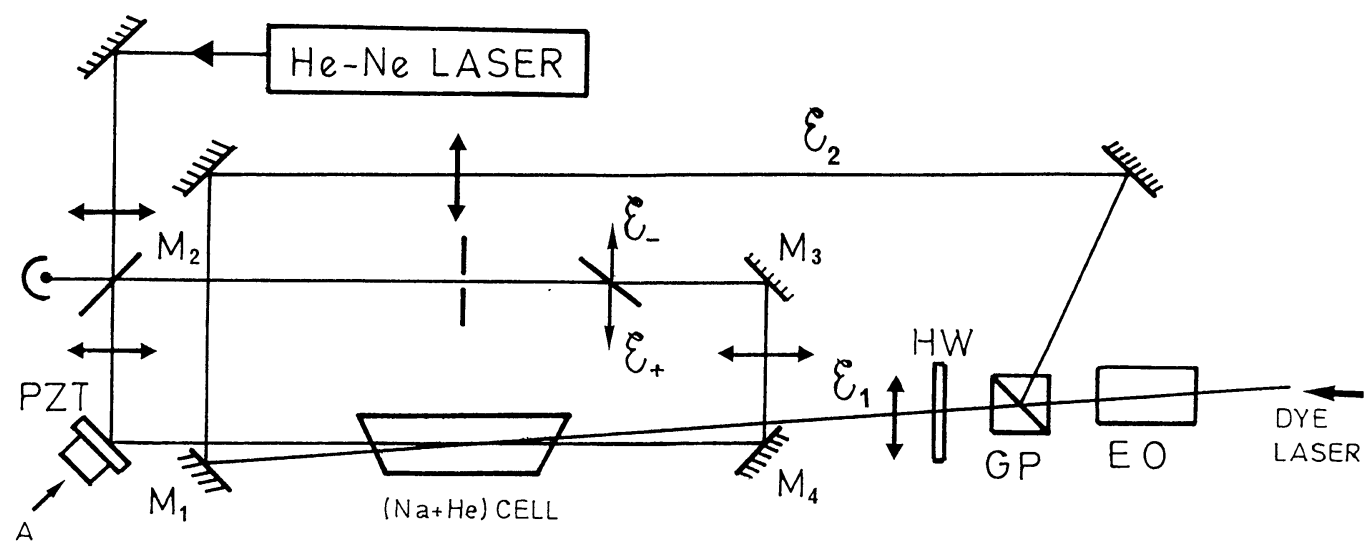

Fig. 4. - Schéma du montage expérimental.

[Experimental set-up.]

$\mathbf{M}_{2}$ qui est partiellement réfléchissant pour le rouge. On détecte l'énergie du faisceau rouge réfléchie par la cavité. On asservit la longueur de la cavité de façon à maintenir l'égalité entre la fréquence du laser $\mathrm{He}-\mathrm{Ne}$ et une des fréquences de résonance de la cavité. Le contrôle de la longueur de la cavité se fait par l'intermédiaire de la cale piézo-électrique (PZT).

Un diaphragme est placé dans la cavité pour éviter une oscillation dans les modes transverses.

La fréquence $\omega$ des ondes pompes est proche de $\omega_{0}$ (fréquence de la raie $\mathrm{D}_{2}$ ), mais l'excitation n'est pas résonante. Le désaccord $\delta$ entre $\omega$ et $\omega_{0}\left(\delta=\omega_{0}-\omega\right)$ est en valeur absolue compris entre $1 \mathrm{GHz}$ et $3 \mathrm{GHz}$. Dans ces conditions on a mesuré la réflectivité par conjugaison de phase $R_{\mathrm{c}}$ pour une onde sonde progressive et on a trouvé que $R_{\mathrm{c}}$ est au plus égale à $1 \%$. On voit donc que le gain $R_{\mathrm{c}}$ est inférieur aux pertes qui sont au moins égales à $15 \%$. Cette valeur de $R_{\mathrm{c}}$ est néanmoins suffisante pour obtenir l'oscillation car le seuil d'un tel oscillateur (voir $\S 3 \mathrm{~Gb}$ ) est égal au carré des pertes pour une cavité accordée $\left(R_{\mathrm{c}} \geqslant \frac{T^{2}}{4}\right)$. Effectivement, en balayant $L$ la longueur de la cavité on observe l'oscillation simultanée de deux ondes contrarotatives $\boldsymbol{\varepsilon}_{+}$et $\boldsymbol{\varepsilon}_{-}$. On constate que l'oscillation ne se produit que pour des valeurs particulières de $L$ (condition de résonance simultanée sur $\omega_{+}$et $\omega_{-}$) et que ces valeurs particulières de $L$ sont équidistantes. On constate aussi que conformément à la théorie, l'intensité de sortie présente un comportement bistable (voir § $3 \mathrm{~Gb}$ ). Enfin on constate que l'intensité $I$ présente deux types de résonance qui diffèrent essentiellement par l'intensité de sortie. En asservissant la longueur de la cavité on a pu étudier en détail les deux modes de fonctionnement de l'oscillateur. Nous ne discuterons dans cet article que les propriétés de mode le plus intense qui correspond à un fonctionnement quasi dégénéré. On a montré que l'autre mode est non dégénéré et que la différence $\omega_{+}-\omega_{-}$est en valeur absolue égale à l'intervalle spectral libre de la cavité [35].

B) ETUDE DU MODE QUASI DÉGÉNÉRÉ.

1) Biais optique. - La valeur typique pour les intensités de sortie $I_{+}$et $I_{-}$est de l'ordre du milliwatt. La fréquence de battement $\Delta \nu=\nu_{+}-\nu_{-}$entre les faisceaux $\varepsilon_{+}$et $\varepsilon_{-}$est mesurée en envoyant le signal de battement dans un analyseur de spectre. La figure 5 montre la variation de $\Delta \nu$ en fonction du paramètre $\left(I_{1}-I_{2}\right) / I_{0}$. Pour des valeurs de ce paramètre 


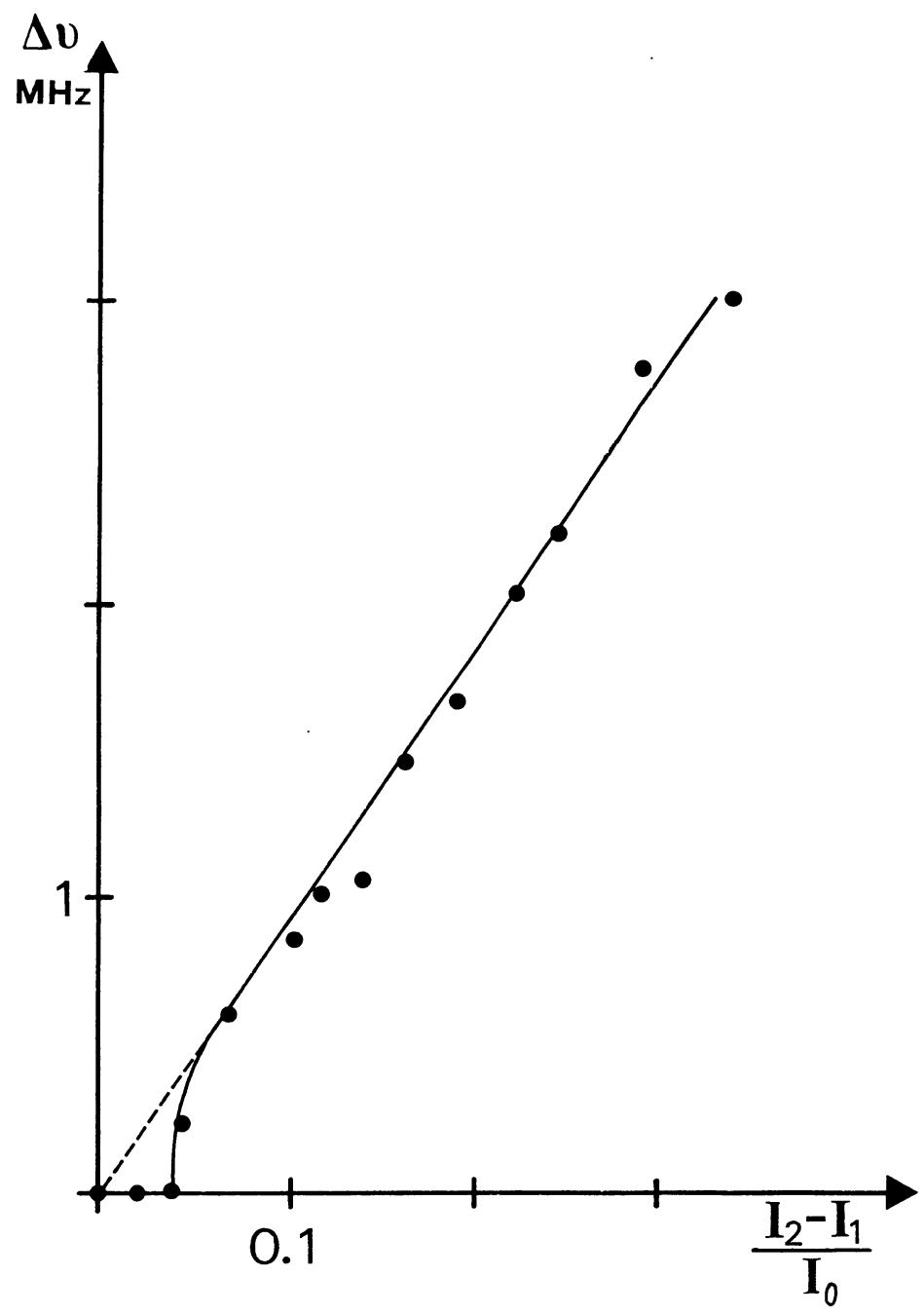

Fig. 5. - Variation de la fréquence de battement $\Delta \nu$ en fonction de la différence d'intensité $\left(I_{2}-I_{1}\right) / I_{0}$ entre les deux ondes pompes ( $2 I_{0}$ est l'intensité totale). L'expérience est réalisée avec une cellule contenant 3 torrs d'hélium.

[Variation of the beat frequency $\Delta \nu$ as a function of $\left(I_{2}-I_{1}\right) / I_{0}$ where $2 I_{0}$ is the constant total intensity. The experiment is done with a cell containing 3 torrs of helium.]

comprises entre 0,1 et 0,3 la fréquence de battement $\Delta \nu$ est bien une fonction linéaire conformément à la théorie. Pour des valeurs plus petites on observe une déviation à la loi linéaire. Ce type de comportement est bien connu dans les gyromètres actifs et correspond à la zone aveugle du gyrolaser [1].

Notre gyromètre a une zone aveugle de $200 \mathrm{kHz}$, ce qui, compte tenu de son facteur d'échelle $S$ [1], correspond à une vitesse de rotation de $50 \%$. Cette valeur est très grande comparée à la zone aveugle des gyrolasers, ceci pouvant être partiellement expliqué par la qualité courante des éléments optiques utilisés dans notre cavité. Chaque point de la figure 5 
est entaché d'une incertitude de $25 \mathrm{kHz}$. Il semble que la position de la fréquence de battement fluctue rapidement conduisant à une largeur apparente de $25 \mathrm{kHz}$. L'origine de cette largeur est à l'heure actuelle incomprise. Il semble que cette largeur soit partiellement due aux fluctuations de l'intensité des ondes oscillantes. En effet lorsque ces fluctuations augmentent la largeur augmente.

Cette largeur peut aussi être due à un déplacement relatif des deux faisceaux pompes entraînant une modification de $\left(I_{1}-I_{2}\right)$ donc du biais. Ce déplacement peut être engendré soit par une imperfection expérimentale soit par des effets spatiaux provenant du milieu non linéaire.

Cet effet lié au caractère gaussien des faisceaux n'a pas été pris en compte dans la théorie développée dans cet article.

$\mathrm{Si}$ ces problèmes étaient résolus on pourrait utiliser cet effet non linéaire non réciproque pour remplacer l'activation mécanique utilisée dans les gyrolasers par une activation optique dans un gyromètre à mélange à quatre ondes.

On pourrait par exemple utiliser deux faisceaux pompes d'intensité $I_{1}=I_{0}+I(t)$ et $I_{2}=I_{0}-I(t)$ où $I(t)$ est une fonction créneau variant entre $-\Delta I$ et $\Delta I$. La différence entre les deux fréquences de battement mesurées dans ces deux conditions nous donnerait directement la fréquence de battement créé par l'effet Sagnac et donc de la vitesse de rotation $\dot{\theta}$ du gyromètre.

Cette méthode d'obtention d'une courbe gyrométrique bien que présentant de nombreux avantages est un peu trop indirecte. C'est pourquoi nous avons décidé de reprendre l'expérience en utilisant un autre processus bien maîtrisé pour créer la non réciprocité. Cette non réciprocité est obtenue en plaçant dans la cavité un rotateur de Faraday.

2) Biais induit par effet Faraday. - Le rotateur de Faraday que nous avons utilisé est constitué d'un barreau de TGG placé dans un champ magnétique B créé par un solénoïde parcouru par un courant $i$. Ce barreau est placé entre deux lames quart d'onde dont les lignes neutres sont orientées à $45^{\circ}$ des axes horizontal et vertical. L'orientation relative de ces deux lames est telle que la polarisation de la lumière reste inchangée après la traversée des deux lames.

La cavité utilisée dans cette expérience est identique à celle utilisée dans l'expérience précédente. En revanche, dans cette expérience, la cellule utilisée est une cellule de sodium pur (sans gaz tampon), chauffée à $170{ }^{\circ} \mathrm{C}$ environ et les deux ondes pompes ont des intensités identiques $\left(I_{1}=I_{2}=I_{0}\right)$. Ces deux ondes sont focalisées dans la cellule sur une tache dont le diamètre est de $300 \mu \mathrm{m}$. Pour un désaccord en fréquence $\delta$ compris en valeur absolue entre $1 \mathrm{GHz}$ et $3 \mathrm{GHz}$ on observe en balayant la cavité une série de résonances fines comme dans l'expérience précédente (\$ 4B.1). En stabilisant la longueur de la cavité on a pu étudier en détail l'influence du rotateur de Faraday sur le mode quasi dégénéré.

La figure 6 montre la variation de la fréquence $\Delta \nu$ de battement entre les deux ondes contrarotatives $\varepsilon_{+}$et $\varepsilon_{-}$émise par l'oscillateur en fonction du courant $i$ circulant dans le solénoïde.

Les points de la figure 6 ont été obtenus pour une valeur $I$ de l'intensité de sortie, les étoiles correspondent à des mesures effectuées pour une intensité moitié. Le fait que les résultats correspondant à des intensités différentes se placent sur la même courbe montre que le facteur d'échelle ne dépend pas du point de fonctionnement de l'oscillateur ce qui est en accord avec les prédictions de la partie théorique.

Ces courbes sont tout à fait similaires à celle obtenue précédemment dans le cas du biais optique. On observe bien un comportement linéaire de la fréquence de battement pour des valeurs suffisantes de $i$. En revanche, pour de faibles valeurs de $i$ on observe une déviation par rapport à la loi linéaire et pour $|i|<I_{\mathrm{c}}$ la fréquence de battement est nulle. 


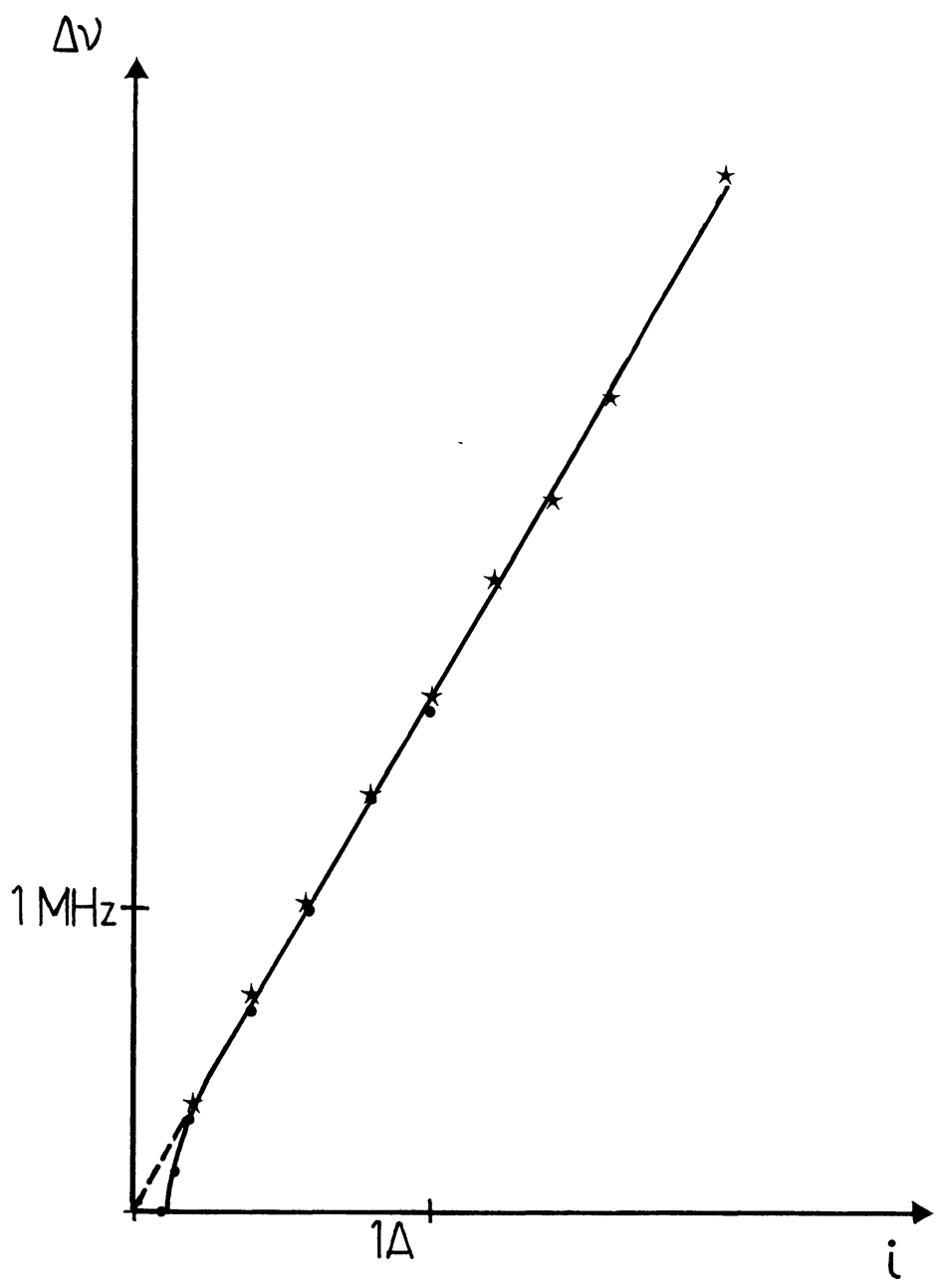

Fig. 6. - Variation de la fréquence de battement $\Delta \nu$ en fonction du courant $i$ circulant dans le rotateur de Faraday. La cellule d'expérience est remplie exclusivement de sodium. Les points ont été obtenus pour une intensité émise $I$, les étoiles pour une intensité $I / 2$.

[Variation of the beat frequency $\Delta \nu$ as a function of the current flowing through the Faraday rotator. The cell only contains sodium. Dots were obtained for an emitted intensity $I$, stars for an intensity $I / 2$.]

La levée de dégénérescence qui est de $1,7 \mathrm{MHz}$ par ampère, lorsque $|i|$ est très grand devant $i_{\mathrm{c}}$, doit être comparée à la valeur prévue théoriquement. En effet, le rotateur de Faraday utilisé crée pour un courant $i$ une levée de dégénérescence $\Delta \nu$ entre les deux ondes contrarotatives égale :

$$
\Delta \nu=\frac{c}{L} \frac{V}{\pi} n i \int_{0}^{d} f(z) \mathrm{d} z
$$

$V$ est la constante de Verdet du barreau utilisé (TGG dans notre expérience) et $d$ sa longueur. $n$ est le nombre de spires par unité de longueur de solénoïde et la fonction $f(z)$ tient compte du trajet parcouru par les faisceaux lumineux. 
Le calcul conduit à un biais égal à $(1,6 \pm 0,2) \mathrm{MHz} / \mathrm{A}$ ce qui est en bon accord avec nos résultats expérimentaux.

Le comportement de la fréquence de battement en fonction du courant $i$ est identique au comportement observé dans l'expérience précédente. En particulier la zone aveugle a une dimension semblable de même que la largeur de battement. Ceci prouve d'ailleurs que la non réciprocité obtenue par le biais optique n'est pas la cause unique de l'élargissement.

\section{Conclusion.}

Nous avons présenté les principales caractéristiques d'un oscillateur en anneau utilisant le mélange à quatre ondes dans une vapeur atomique comme processus de gain. Nous avons limité notre étude au mode de fonctionnement quasi dégénéré. Nous avons montré théoriquement que dans ce mode de fonctionnement l'oscillateur à mélange à quatre ondes se comporte comme un gyromètre optique actif : son facteur d'échelle est identique à celui des gyrolasers et sa sensibilité est aussi limitée par le processus de rétrodiffusion qui est responsable de la zone aveugle du gyromètre.

Nous avons montré que l'utilisation d'une non-linéarité intrinsèque permet par un moyen purement optique de s'affranchir de la zone aveugle. Nous avons mis en évidence expérimentalement l'ensemble de ces propriétés gyrométriques en utilisant une vapeur de sodium interagissant avec deux ondes pompes contrapropagatives de fréquence proche de celle de la raie $D_{2}\left(3 S_{1 / 2} \rightarrow 3 P_{3 / 2}\right)$.

Plusieurs extensions sont possibles pour ce travail. Premièrement notre théorie est valable uniquement au voisinage du seuil car nous avons supposé que l'énergie prélevée aux pompes était très faible. Une théorie valable pour une intensité quelconque serait évidemment intéressante pour comprendre le comportement de ce gyromètre à forte intensité. Deuxièmement le modèle atomes immobiles et le fait que nous négligeons l'absorption est certainement criticable car le désaccord à la résonance n'est pas suffisamment grand devant la largeur Doppler pour éliminer totalement l'influence des classes de vitesse résonnantes. Néanmoins, en dépit de ces restrictions, le modèle présenté ici permet d'expliquer la majorité des résultats obtenus expérimentalement.

Du point de vue expérimental, l'utilisation d'un laser à colorant pompé par un laser à argon ionisé peut sembler un obstacle rédhibitoire au développement de tels gyromètres. En fait, une miniaturisation de notre système est parfaitement envisageable en utilisant un alcalin plus lourd et des diodes lasers [36]. Néanmoins, toute démarche dans cette direction doit être précédée par une meilleure compréhension de l'origine de la largeur de la courbe de battement. Il nous est apparu qu'un des paramètres influant de manière significative sur la forme de la courbe de battement est l'angle et le recouvrement des ondes pompes. Ceci suggère que les problèmes spatiaux, tels que la définition des modes transverses pour un tel oscillateur, mériteraient d'être considérés dans le prolongement de cette étude.

\section{Remerciements.}

Les auteurs tiennent à remercier la DRET pour son concours à cette étude. Ils remercient également M. Faucheux et J. J. Roland de Quantel pour les nombreuses et fructueuses discussions sur cette expérience.

\section{Bibliographie}

[1] Hellwarth R. W., J. Opt. Soc. Am. 67 (1977) 1; YARIV A. and PePPER D. M., Opt. Lett. 1 (1977) 16 ; see also Optical Phase conjugation, edited by R. Fisher (Academic, New York, 1983). 
[2] Yuen H. P. and Shapiro J. H., Opt. Lett. 4 (1979) 334.

[3] Walls D. F., Nature (London) 306 (1983) 141.

[4] Slusher R. E., Holberg L. W., Mertz J. C. and Valley J. F., Phys. Rev. Lett. 55 (1985) 2409 ; SLUSHER R. E. and YURKe B., in Frontiers in Quantum Optics, edited by S. Sarkar and E. R. Pike (Hilger, London, 1986).

[5] Shelby R. M., Levenson M. D., Perlmutter S. H., De Voe R. G., Walls D. F., Phys. Rev. Lett. 57 (1986) 691 ;

Ling au Wu, Kimble H. J., Hall J. L. and Huifa Wu, Phys. Rev. Lett. 57 (1986) 2520.

[6] Maeda M. W., Kumar P. and Shapiro J. H., Phys. Rev. A 32 (1985) 3803.

[7] Vallet M., Pinard M. and Grynberg G., Europhys. Lett., 11 (1990) 739.

[8] LiNd R. C. and Steel D. G., Opt. Lett. 6 (1981) 554 ;

Kleinmann B., Trehin F., Pinard M. and Grynberg G., J. Opt. Soc. Am. B 2 (1985) 704 ;

Le Bihan E., Pinard M. and Grynberg G., Opt. Lett. 11 (1986) 159 ;

Leite J. R., Simoneau P., Bloch D., LeboiteuX S. and Ducloy M., Europhys. Lett. 2 (1986) 749.

[9] White J. O., Cronin-Colomb M., Fisher B. and Yariv A., Appl. Phys. Lett. 40 (1982) 450 ; Yariv A. and Sze-Keung KwONG, Opt. Lett. 10 (1985) 454 ;

Mac Donald K. R. and Feinberg J., J. Opt. Soc. Am. 1 (1984) 1213 and Phys. Rev. Lett. 55 (1985) 821 ;

Rajbenbach H. and Huignard J. P., Opt. Lett. 10 (1985) 137.

[10] Feinberg J. and Bacher G. D., Opt. Lett. 9 (1984) 420.

[11] Mac Donald K. R. and Feinberg J., J. Opt. Soc. Amer. A 1 (1984) 1213.

[12] Mac Donald K. R. and Feinberg J., Phys. Rev. Lett. 55 (1985) 821.

[13] Rajbenbach H. and Huignard J. P., Opt. Lett. 10 (1985) 137.

[14] Yariv A. and Kwong S. K., Opt. Lett. 10 (1985) 454.

[15] Pinard M., Grandclement D. and Grynberg G., Europhys. Lett. 2 (1986) 755.

[16] Grandclement D., Grynberg G. and Pinard M., Phys. Rev. Lett. 59 (1987) 44.

[17] Grandclement D., Pinard M. and Grynberg G., IEEE J. Quantum Electron. 25 (1989) 580.

[18] Pinard M., Horowicz R., Grandclement D. and Grynberg G., IEEE J. Quantum Electron. 25 (1989) 570.

[19] Shen Y. R., "The principle of Non-linear Optics" (New York : Wiley (1984) ch. 9-2).

[20] Murray Sargent III, Scully M. O., Lamb W. E. Jr., "Laser Physics » Addison-Wesley Publishing Company (1974).

[21] LeFloch A., Communication privée Gyromètres passifs résonants.

[22] Sagnac G., C.R. Hebd. Acad. Sci. Paris 95 (1913) 708.

[23] Chow W. W. et Coll. Rev. of Modern Physics 57 (1985) 61.

[24] Faucheux M., Fayoux D., Roland J. J., J. Optics 19 (1988) 101.

[25] Bloembergen N., Bogdan A. R. and Downer M. W., in Laser Spectroscopy V edited by Mc

Kellar A. R. W., Oka T. and Stroicheff B. P. (Springer, Heidelberg) (1981) 157.

[26] Grynberg G. and Pinard M., Phys. Rev. A 32 (1985) 3772.

[27] Bloch D., Thèse de $3^{\mathrm{e}}$ cycle, Université Paris XI (1980).

[28] Grynberg G., Le Bihan E. and Pinard M., J. Phys. France 47 (1986) 1321 ;

GrandClement D., Grynberg G. and Pinard M., Phys. Rev. Lett. 59 (1987) $40 ; 59$ (1987) 40.

[29] Grynberg G. and Pinard M., C.R. Hebd. Acad. Sci. Paris 306 (1988) 203 ;

Grynberg G., Pinard M., Faucheux M., Opt. Lett. 14 (1989) 63.

[30] Gibbs H. M., Mac Call S. L. and Venkatesan T. N. C., Phys. Rev. Lett. 36 (1976) 1135.

[31] GibBS H. M., Optical Bistability : Controlling Light with Light. New York: Academic (1985).

[32] Giacobino E., Devaud M., Biraben F. and Grynberg G., Phys. Rev. Lett. 45 (1980) 434.

[33] AdLer R., Proceedings of the IEEE 61 (1973) $\mathrm{n}^{\circ} 10$.

[34] Cresser J. D., Louisell W. H., Meystre P., Schleich W. and Scully M. O., Phys. Rev. A 25 (1982) 2214.

[35] Grynberg G., Pinard M. and Verkerk P., Europhy's. Lett. 9 (1989) 139.

[36] Oria M., Bloch D., Fichet M. and Ducloy M., Opt. Le't. 19 (1989) 1082. 